\title{
Review of the "City Health Profiles" produced by WHO-Healthy Cities-do they present information on health and its determinants and what are their perceived benefits?
}

\author{
Premila Webster
}

The Healthy Cities Project (HCP) builds on the WHO definition of health as a state of complete physical, mental, and social well being and has become an important WHO vehicle for promoting Health For All strategy at a local level. ${ }^{1-3}$ One of the requirements to be designated a "Healthy City" is to produce a City Health Profile (CHP), bringing together key information on health and its determinants. This would form the basis of a City Health $\mathrm{Plan}^{4}$ setting out strategies and intervention programmes to improve the population's health.

The WHO publication "City Health Profiles: how to report on health in your city" (produced by the WHO-CHP Technical Group following consultations with primary users) offered guidance on producing profiles.

In 1996 a review of 21 CHPs was undertaken to examine whether they described health and its determinants. As CHPs were a requirement to be designated a "healthy city" the review also examined whether the Healthy Cities coordinators observed any benefits other than fulfilling the requirement for designation.

\section{Methods}

The methods used were (1) a structured, systematic "content" review by two reviewers and (2) complemented by semi-structured telephone interviews.

The criteria outlined in the WHO document $^{5}$ were the benchmark used to compare the description of health and its determinants and formed the framework for the content review. Nine main categories were examined. Each of the categories and subcategories were coded as having no, some and detailed information (indicating a qualitative assessment of depth rather than a quantitative assessment of length). Zero, one and two points respectively were awarded.

A semi-structured questionnaire was developed to obtain additional information on the contents, process, and perceived benefits. This was piloted in three cities, one English mother tongue and two non-English mother tongue including one city from Eastern Europe. Representatives (the coordinator of the HCP
Table 1 Coverage of countries in the profiles reviewed

\begin{tabular}{ll}
\hline Country & Number \\
\hline Austria & 1 \\
Czech Republic & 1 \\
Denmark & 2 \\
Germany & 1 \\
Hungary & 1 \\
Ireland & 1 \\
Israel & 1 \\
Italy & 1 \\
Netherlands & 2 \\
Norway & 1 \\
Poland & 2 \\
Portugal & 1 \\
Slovenia & 1 \\
Switzerland & 1 \\
UK & 4
\end{tabular}

or a person nominated by them) from all the cities were interviewed by telephone.

\section{Results}

The profiles were drawn from 15 European countries (table 1). The level of agreement between the two reviewers was measured by $\kappa$, 0.83 showing very good strength of agreement. The areas of disagreement were exclusively between "yes" and "detailed", they were reviewed and consensus reached. Data collected are presented in table 2.

Table 2 Coverage of health and its determinants in the profiles reviewed

\begin{tabular}{lcrr}
\hline Category & Detailed & Yes & No \\
\hline Demography & 100 & & \\
Mortality & 100 & & \\
Morbidity & 67 & 19 & 14 \\
$\quad$ Health services & 28 & 48 & 24 \\
$\quad$ Primary care & 24 & 5 & 71 \\
$\quad$ Perceived health & & & \\
Lifestyle & 33 & 24 & 43 \\
$\quad$ Smoking & 29 & 42 & 29 \\
$\quad$ Alcohol & 29 & 29 & 42 \\
$\quad$ Drugs & 10 & 38 & 52 \\
$\quad$ Diet & 29 & 33 & 38 \\
$\quad$ Exercise & & & \\
SE conditions & 48 & 30 & 22 \\
$\quad$ Housing & 33 & 22 & 45 \\
$\quad$ Education & 38 & 38 & 24 \\
$\quad$ Employment & 30 & 22 & 48 \\
$\quad$ Crime & 8 & 48 & 44 \\
$\quad$ Income & 95 & 5 & 0 \\
Physical environment & 29 & 38 & 33 \\
Inequalities & 14 & 34 & 52 \\
Infrastructure & 33 & 38 & 29 \\
PH policies and structures & &
\end{tabular}

Data shown as percentages. 
HEALTH INFORMATION

The scores ranged between 6 and 37 out of a maximum 38 (mean 18.8, median 18, mode 23, (SD 7.37)), with $23 \%$ in the lower $(<14)$, $29 \%$ median (14-22) and $48 \%$ upper quartiles $(>22)$. Three distinct patterns emerged:

(1) Profiles in the lower quartile described "health" in terms of data on demography and major mortality and morbidity. Several had figures on hospital service utilisation and basic data on "the environment" (that is, information on air and water quality and waste disposal). Most echoed forms of statistical returns obtained from statutory data collecting bodies. Very limited or no data were available on perceived health status, lifestyles, socioeconomic conditions, inequalities (which ascertained whether data from other sections were analysed to draw attention to inequalities in health) and public health policies and structures in the profiles in this quartile.

(2) Profiles in the median quartile enriched the basic statistics by incorporating data from local surveys including life style surveys. Several of them had information on housing, employment, and transport and on some public health policies and structures. Information on inequalities was limited, the data presented being mainly on the higher mortality rates among certain ethnic groups and homeless people.

(3) Profiles in the upper quartile had consolidated data collected from several agencies and included information from relevant local surveys, presenting a holistic picture of the citizen's health. Some had detailed information on inequalities, including analysis down to small area levels.

THE PROCESS OF PRODUCING THE PROFILES

In the planning stages all cities had identified a lead agency (for two thirds it was the HCP and for the rest the academic department of either public health or epidemiology). In $29 \%$ of the cities sponsors financed the profile, $71 \%$ used the HCP budget. It was difficult to get costing details, as in several cities resources, both intellectual and otherwise had been offered free of charge (for example academics had given their services free, printers had not charged, etc). Target audiences were identified by $65 \%$ of the cities, general content decided and data sources identified by $72 \%$. Though all had set timetables only $42 \%$ had managed to meet the deadline.

All cities that produced profiles following the publication of the WHO guidelines used it as a framework. Profiles produced before these guidelines used other city's profiles as a guide or frameworks developed with the help of their academic institutions. All cities had at least one professional with expertise in the technical aspects of health data collection responsible for checking the validity and robustness of the data.

Eighty six per cent of the cities obtained media coverage to publicise their profiles. Forty eight per cent had meetings with local politicians to present their profiles. Seventy one per cent organised a public launch of the profile that was often inaugurated by the city mayor or a local politician.

\section{PERCEIVED BENEFITS}

Data from interviews were grouped into descriptive categories and analysed. Three principal benefits emerged.

(1) All observed that profiles provided important baseline information on health and proved to be vital planning instruments.

(2) Eighty six per cent noticed that by providing information based on robust data, profiles gave them the opportunity and leverage to motivate politicians to tackle areas of need.

(3) Seventy one per cent said the process of producing profiles involved players from different disciplines, which had provided the opportunity to build alliances with agencies involved in influencing health.

\section{Discussion}

Health profiles are a common form of presenting health information, however, little work has been undertaken in analysing their content, process or usefulness. Though this study was only on 21 profiles, they were drawn from different countries with varied cultures, political systems and contrasting philosophies on health and social welfare. It is also one of very few studies that has attempted to undertake a systematic and comprehensive content review by expanding the "text approach" with telephone interviews. The findings indicate that profiles presenting information on health and its determinants rather than illness and hospital data alone do describe "health". It provided some understanding of the process involved and suggested that profiles could have some influence in placing health on the agenda of politicians and encourage multi-agency cooperation to build health alliances.

In designing the review a considerable degree of effort was expended to ensure the reliability and validity of the data collected. The use of a structured format and two independent reviewers was aimed at making the review objective. There was a high inter-rater agreement. However, the review only coded the content as being absent, present or detailed, what this missed was the degree of detail that was available. However, this may have made the review process complex and difficult to analyse.

A telephone questionnaire was thought to be the best option to collect the additional information and it was piloted in three cities before use. Though this allowed information to be collected from all cities it was not anonymous and meant that the respondents may have given socially desirable answers and could be a possible source of bias. To minimise bias, respondents were assured that this was not a ranking exercise, no information could be linked to the respondent and data presented would be anonymous.

Following the review the WHO-HCP Office set up workshops to explore the results with cities and identify areas where support was required. Experts in the field evaluated the sec- 
tions on lifestyles and inequalities (where cities were experiencing the most problems) in greater depth and developed guidelines. ${ }^{6}$

Organisation, meticulous planning, and a sound framework are required to produce profiles that accurately reflect the population's health. It entails working with appropriate agencies influencing health to gather relevant, valid, and reliable information, presenting it in a suitable format and ensuring wide dissemination if it is to work as a reliable instrument in health planning.

I wish to thank Dr Agis Tsouros and the WHO-Healthy Cities Project Office, Copenhagen for providing me with the opportunity and resources to undertake this work, Dr Charles Price for advice and support in setting up the project, the WHO Profiles and Indicators Technical Group and their chair Dr Mark McCarthy for technical advice and support and all the coordinators of the European WHO-HCP without whose help and cooperation this work would not have been possible.

1 Kickbusch I. Healthy cities a working project and a growing movement. Health Promotion 1989;4:77-82.

2 Scally G. Citizen health. [Editorial]. Lancet 1996;347:3-4.

3 Tsouros AD. The WHO Healthy Cities Project: state of the art and future plans. Health Promotion 1995;10:133-41.

4 WHO Healthy City Project Office. City health planning: the framework. World Health Organisation Regional Office for Europe, 1996;EUR/ICP/HCIT 94 01/MT06/7.

5 WHO Healthy City Project Technical Working Group on City Health Profiles. City health profiles: how to report on health in your city. World Health Organisation Regional Office for Europe, 1995;ICP/HSIT/94/01 PB 02.

6 Acres J. Using city health profiles to reduce inequity in health. In: City health profiles- a review of progress. Copenhagen: World Health Organisation Regional Office for Europe, 1998:9-20.

7 Rasmussen NK. Lifestyles in city health profiles. In: City health profiles-a review of progress. Copenhagen: World health profiles-a review of progress. Copenhagen: World 38. 